\title{
Solar models and electron screening
}

\author{
A. Weiss ${ }^{1,2,3}$, M. Flaskamp ${ }^{1}$, and V. N. Tsytovich ${ }^{4}$ \\ 1 Max-Planck-Institut für Astrophysik, Karl-Schwarzschild-Str. 1, 85748 Garching, Germany \\ 2 Princeton University Observatory, Peyton Hall, Princeton, NJ 08540, USA \\ 3 Institute for Advanced Study, Einstein Dr., Princeton, NJ 08540, USA \\ 4 Dept. of Theoretical Physics, General Physics Institute, Russian Academy of Sciences, Moscow, Russia
}

Received 14 December 2000 / Accepted 6 February 2001

\begin{abstract}
We investigate the sensitivity of the solar model to changes in the nuclear reaction screening factors. We show that the sound speed profile as determined by helioseismology certainly rules out changes in the screening factors exceeding more than $10 \%$. A slightly improved solar model could be obtained by enhancing screening by about 5\% over the Salpeter value. We also discuss how envelope properties of the Sun depend on screening, too. We conclude that the solar model can be used to help settling the on-going dispute about the "correct" screening factors.
\end{abstract}

Key words. nuclear reactions - plasmas - Sun: general - interior

\section{Introduction}

The solar interior is, thanks to the tremendous progress of helioseismology, known to such great accuracy that the Sun can in fact be used as a laboratory for physics. Examples are investigations about the equation of state (Däppen \& Nayfonov 2000) or axion properties (Schlattl et al. 1999). In this paper, we will apply it to the Coulomb screening of the nuclear reactions.

The plasma correction to nuclear reaction rates, also known as screening, is one of the ingredients for stellar and solar model calculations, which has repeatedly been rediscussed in the literature. The standard derivation by Salpeter (1954) discusses electrostatic screening in the Debye-approximation, where the electrostatic potential around an ion of charge $Z_{1} e$ is

$\Phi=\left(Z_{1} e / r\right) \exp (-r / D)$,

where $D$ is the Debye radius $\left(D=k T / 4 \pi e^{2} n_{\mathrm{e}} ; n_{\mathrm{e}}\right.$ : electron density), within which $(r \ll D)$ the electrostatic potential around $Z_{1}$ is reduced to

$\Phi=Z_{1} e / r-Z_{1} e / D$.

This reduced Coulomb potential gives rise to an increased reaction rate by a factor

$f=\exp \left(\frac{Z_{1} Z_{2} e^{2}}{k T D}\right) \approx 1+f_{\mathrm{S}}=1+0.188 Z_{1} Z_{2} \xi^{\frac{1}{2}} \rho^{\frac{1}{2}} T_{6}^{-\frac{3}{2}},(3)$

Send offprint requests to: A. Weiss,

e-mail: weiss@mpa-garching.mpg.de
$Z_{2} e$ denoting the partner charge in the nuclear reaction, and $\xi=\sum_{Z} \frac{\left(Z^{2}+Z\right) X_{Z}}{A_{Z}}$ the effective charge.

Equation (3) has been challenged in various papers (e.g. Carraro et al. 1988; Shaviv \& Shaviv 1996, 2000; Savchenko 1999), either developing different pictures for the configuration of $Z_{2}$ around $Z_{1}$, or by pointing out the static character of Salpeter's derivation, asking for a dynamical one, since we are dealing with a plasma. All these papers have been refuted by other work pointing out flaws, inconsistencies or by re-derivation of Eq. (3) (e.g. Brueggen \& Gough 1997; Gruzinov \& Bahcall 1998). Recently, Bahcall et al. (2000a) have summarized five different derivations of the plasma correction to nuclear reaction rates at the centre of the Sun, all arriving at the Salpeter formula. At the same time they review several papers with deviant screening rates, stressing that all these papers arrive at different factors. In their summary, Bahcall et al. (2000a) emphasize that in the future proving the correctness of screening formulae should be part of the corresponding work. Among the work criticised was also that of one of the present authors (Tsytovich \& Bornatici 2000; Tsytovich 2000), in which screening results in a suppression of nuclear reaction rates in the Sun.

Since many of the paper in this field are not easy to understand and in particular their correctness not easy to be verified nor falsified, we used in Flaskamp et al. (2000) a different approach. Following our previous applications of the Garching Solar Model (GARSOM; Schlattl 1999) as a highly sensitive laboratory for stellar and particle physics (e.g. Schlattl 1999; Schlattl et al. 1999) we 
applied the screening rates of Tsytovich (2000) to our solar model and compared the resulting sound speed with a model with Salpeter's equation. In this preliminary work we reported about changes in sound speed both at the solar centre and in the radiative regions around $r / R_{\odot} \approx 0.65$ ( $R_{\odot}$ is the solar radius), which are inconsistent with helioseismological results.

In the present paper we are extending our analysis to general modifications of Eq. (3) and repeat it for a slightly modified derivation of the screening compared to the original work by Tsytovich \& Bornatici (2000). In Sect. 2 we will briefly summarize our solar models and describe the modified screening values. In Sect. 3 we show the influence on sound speed, neutrino rates and global solar properties and demonstrate that, given the accuracy of the helioseismologically inferred sound speed, even the case of reactions rates without screening $(f=1)$ can be excluded $^{1}$. We then turn around our approach to show that a slightly improved solar model can be obtained by increasing Salpeter's screening by a moderate amount $(\approx 5 \%)$. Finally, in the last section, we conclude that the present work demonstrates convincingly that the Sun can be used as a laboratory for nuclear reaction screening; this could be helpful in the future to settle the on-going dispute about screening.

\section{Calculations and models}

\subsection{Solar models}

We calculated solar models with the program and input physics as described in Schlattl (1999) or Schlattl et al. (1999). The physics is very similar to other standard models such as published recently by Bahcall et al. (2000b). We avoid repeating details here, but emphasize that helium and metal diffusion are included and the present solar luminosity, radius and surface value of $Z / X=0.0245$ are matched (note that Bahcall et al. 2000b use a slightly lower value of 0.0230; Grevesse \& Sauval 1998). The resulting model parameters (Table 1) and sound profile (Fig. 1; solid line) agree well with Bahcall et al. (2000b).

For our standard model ("GARSOM") we have used Salpeter's formula. In all other models, screening factors were replaced as described next. Otherwise the model calculations are identical.

\subsection{Screening factors}

Except for the Tsytovich-screening model (Fig. 1; dashed line), the screening factor $f$ of all reactions of pp-chains and CNO-cycle was multiplied by a constant factor $g$, ranging from 0.9 to 1.1 , such that $f=\left(1+f_{\mathrm{S}}\right) \cdot g$ for the models shown in Fig. 2, or was replaced by $f=1$ for the no-screening model (Fig. 1; dash-dotted line).

For the Tsytovich-screening model a varying screening factor depending on reaction and composition was used

\footnotetext{
${ }^{1}$ We note that a very similar investigation has recently and independently been published by Fiorentini et al. (2000).
}

for the $\mathrm{p}+\mathrm{p},{ }^{3} \mathrm{He}+{ }^{3} \mathrm{He},{ }^{3} \mathrm{He}+{ }^{4} \mathrm{He},{ }^{7} \mathrm{Be}+\mathrm{p}$, and ${ }^{7} \mathrm{Be}+\mathrm{e}^{-}$ reactions. (For all other reactions standard Salpeter values were used.) These factors were determined by starting out with the formulae given in Tsytovich \& Bornatici (2000), which involve the solution of multi-dimensional integrals, which depend on temperature, density, and abundances of the participating nuclei. Instead of evaluating these integrals at all points within the solar model, we did so for a selected number of mass coordinates for several models along a standard model evolution sequence and found that to very good approximation the screening factors can be expressed as a function of helium content (as a parameter describing both the spatial coordinate and the evolution with time within the energy generating core). In Eq. (3) we replace $Z_{1} Z_{2} \xi^{\frac{1}{2}}$ by $F \cdot L_{i j}$, where $F$ is depending on composition and $L_{i j}$ on reaction $i j$. For the centre of the Sun we recover the screening factors given in Table 1 of Tsytovich (2000) when using $F$ and $L_{i j}$ from Tsytovich \& Bornatici (2000).

We note that the original results of Tsytovich \& Bornatici (2000) have been modified slightly by V. N. Tsytovich until the time of the computations presented here and are subject to further ongoing research. With respect to the original paper, the quantity $L_{i j}$ became temperature-dependent (except for $\mathrm{e}^{-}$-capture on ${ }^{7} \mathrm{Be}$ ) and some additional terms in expansion series had been added. Meanwhile the formalism has been improved to treat two-particle distribution functions, a sign-error in one term has been corrected for, and it appears that in addition to the dynamical screening terms the static ones, which correspond to the Salpeter-screening and which canceled in the original derivation (Tsytovich \& Bornatici 2000) could remain, depending on whether plasma perturbations induced by the nuclear reactions themselves can decay in time.

The new derivation and results will be published in a forthcoming paper (Tsytovich 2001, in preparation). We therefore emphasize that the purpose of the present paper is not to investigate the Tsytovich (2000) screening rates, because they are already under further development, but to demonstrate the capability of the Sun as a laboratory for screening using the screening formulation by Tsytovich (2000) as an example.

\section{Results}

\subsection{Sound speed}

The results of our experiments are displayed in Figs. 1 and 2 for the sound speed difference between models $\left(c_{\mathrm{m}}\right)$ and the seismic Sun $\left(c_{\mathrm{s}}\right)$. The inferred sound speed profile we took from Basu (1998). For the error range of $c_{\mathrm{s}}$ we show two different results: the extremely conservative estimation by Degl'Innocenti et al. (1997), shown as the greyshaded area in Fig. 1, and a more recent analysis about the uncertainty of the inversion procedure (Basu et al. 2000). In the latter paper use of SOHO-results was made and the authors concluded that the relative sound speed 


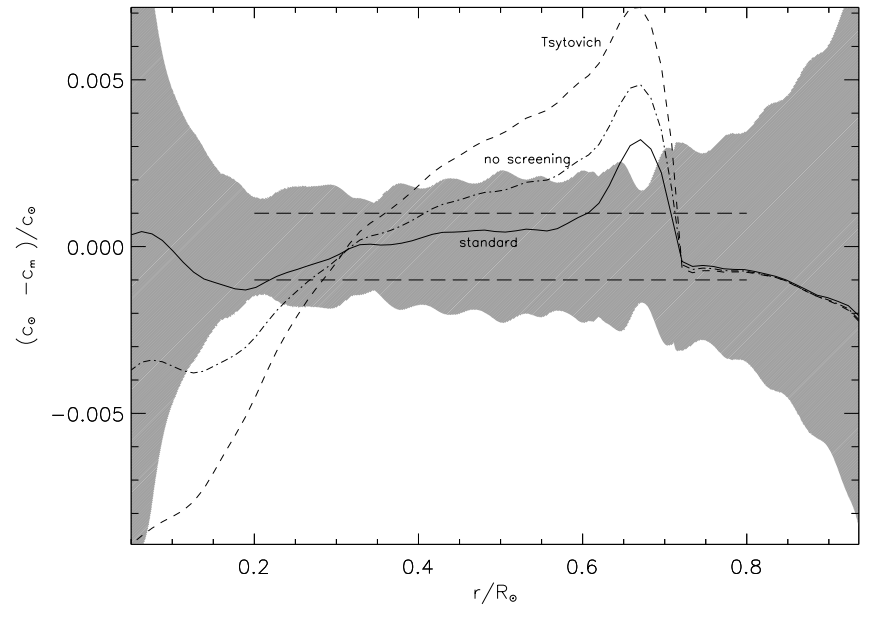

Fig. 1. Comparison of the sound speed profile for our three models using Salpeter- (solid line), Tsytovich- (dashed) and no screening (dash-dotted) with the sound speed derived from helioseismology (see text). The shaded area and the long-dashed line refer to different error estimates in the latter

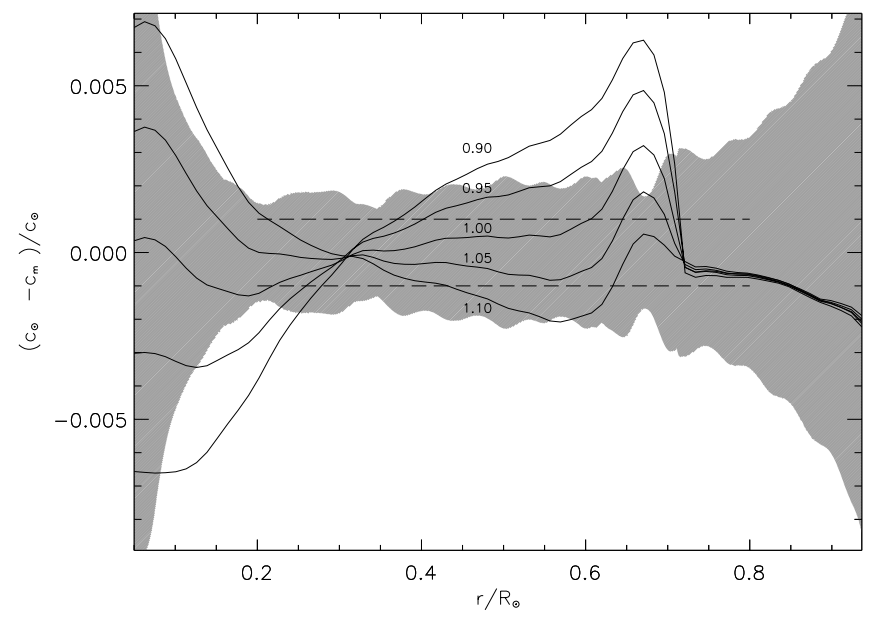

Fig. 2. As Fig. 1, but for models with the Salpeter screening factor $f=1+f_{\mathrm{S}}$ (Eq. (3)) multiplied by a constant factor indicated along the various lines

errors due to the measurements are of order $310^{-4}(3 \sigma)$ and that inversion method and starting model add about equally, thus that a conservative error range based on this paper would be of order $110^{-3}$ for $0.2<r / R_{\odot}<0.8$. This is indicated by the long-dashed line in both figures.

It is immediately clear from Fig. 1 that the model with Tsytovich-screening is stronly discrepant with the seismic model and even the no-screening model is outside the conservative error range for small radii and below the convective envelope. The deviation from the standard model is of order the conservative error range or larger, such that it can be excluded. This is in particular true for the more up-to-date error estimate by Basu et al. (2000) in the radiative interior.

It is interesting to note that going from Salpeterto Tsytovich-screening there are systematic changes in both the core and sub-convective regions, which are anticorrelated. The model's sound speed is increasing in the core due to the higher central temperatures necessary to provide the same number of pp-reactions per second (the solar luminosity constraint). At the same time $c_{\mathrm{m}}$ is decreasing in that part of the radiative zone outside the energy-generating core (extending to $r / R_{\odot} \approx 0.2$ ) due to a slightly more expanded structure. The Sun appears to be more centrally concentrated than it is for standard screening. Recognizing this it is only a small step to ask if the two extrema in Fig. 1 along the standard-model can be reduced simultaneously by increasing the screening above the Salpeter value.

The result of the corresponding experiment is shown in Fig. 2 for different factors multiplied to Eq. (3) for all reactions. An increase of the screening can indeed improve the situation at the discrepant bump around $r / R_{\odot} \approx 0.65$, but leads to a comparatively large change in the core. An increase over Salpeter's screening by more than $10 \%$ will become problematic even in the case of the conservative error estimates; reductions by $5 \%$ or more can clearly be excluded. There are two lessons to be drawn from this:

1. agreement with the seismic sound speed profile is obtained from screening factors not deviating by more than $+10 /-5 \%$ from Salpeter's formula;

2. the discrepant region below the convective envelope might not necessarily be due to missing physics only in that region; it should not be forgotten that the Sun is a system coupling different regions and that changes in the core also affect the rest of the Sun.

We close this section by reminding that the structural changes just discussed depend to first order only on the screening of the pp-reaction (as we have verified in test calculations), which is the one directly connected to the solar luminosity. All statements made above therefore restrict the screening of only this reaction. We also remind the reader that the reaction rate itself is influencing the model as shown by Antia \& Chitre (1999); we are using the rate by Adelberger et al. (1998), where $S(0)=4.0010^{-25} \mathrm{MeVb}$. This value, together with that of the central metallicity $\left(Z_{\mathrm{c}}=0.021\right)$ is marginally consistent with helioseismic data, as shown in Antia \& Chitre (1999), Fig. 2.

\subsection{Other properties}

The Sun does not only show the influence of screening in its sound speed profile, but also in some other properties (Table 1; lower part). Related to results based on helioseismology are the depth of the convective envelope and the present surface helium content, for which the determined values are $R_{\mathrm{bcz}} / R_{\odot}=0.713 \pm 0.001$ (Basu \& Antia 1997) and $Y_{\mathrm{s}}=0.249 \pm 0.003$ (Basu \& Antia 1995). While the Salpeter-model nicely fits, the no-screening case already is at the border of the error range and the Tsytovich-model disagrees with the value for $R_{\mathrm{bcz}}$ and is only marginally consistent with that for $Y_{\mathrm{s}}$. Note that these are envelope resp. surface quantities, but the change to the physics is done deep inside the core! 
Table 1. Properties of the three solar models of Fig. 1. In the upper part neutrino fluxes (in $\mathrm{s}^{-1} \mathrm{~cm}^{-2}$ ) as predicted from the models are given. In the central part the corresponding expected measurements in the three experiments are listed; the measured values are given in the first column. For the Cland Ga-experiments the unit is in SNU, for Super-Kamiokande it is in units of the standard model prediction. The lower part shows some global properties of the initial and present Sun. Note that our mixing-length parameter is different from those of other standard models because of the different treatment of convection and atmospheres in our models (see Schlattl 1999)

\begin{tabular}{l|r|r|r}
\hline & Salpeter & no screening & Tsytovich \\
\hline $\mathrm{pp}$ & $5.9310^{10}$ & $5.9610^{10}$ & $5.9610^{10}$ \\
pep & $1.39010^{8}$ & $1.42710^{8}$ & $1.46310^{8}$ \\
$\mathrm{hep}$ & $2.07610^{3}$ & $2.10710^{3}$ & $2.45610^{3}$ \\
$7 \mathrm{Be}$ & $4.81210^{9}$ & $4.67710^{9}$ & $4.02310^{9}$ \\
$8 \mathrm{~B}$ & $5.05410^{6}$ & $5.35310^{6}$ & $5.22510^{6}$ \\
$13 \mathrm{~N}$ & $5.84910^{8}$ & $5.00410^{8}$ & $8.10110^{8}$ \\
$15 \mathrm{O}$ & $5.07210^{8}$ & $4.20610^{8}$ & $7.32310^{8}$ \\
$17 \mathrm{~F}$ & $6.25110^{6}$ & $5.01710^{6}$ & $9.20010^{6}$ \\
\hline $\mathrm{Sum}$ & $6.5410^{10}$ & $6.5410^{10}$ & $6.5410^{10}$ \\
\hline $\mathrm{Cl}(2.56)$ & 7.579 & 7.819 & 7.790 \\
$\mathrm{Ga}(72.5)$ & 128.40 & 127.05 & 127.61 \\
$\mathrm{SK}(47.8 \%)$ & $100 \%$ & $105.9 \%$ & $103.4 \%$ \\
\hline$\alpha$ & 0.9750 & 0.9281 & 0.8731 \\
$Y_{i}$ & 0.2747 & 0.2734 & 0.2721 \\
$Z_{i}$ & 0.0199 & 0.0200 & 0.0201 \\
$Y_{\mathrm{s}}$ & 0.2448 & 0.2428 & 0.2403 \\
$Z_{\mathrm{s}}$ & 0.0181 & 0.0181 & 0.0182 \\
$R_{\mathrm{bcz}} / R_{\odot}$ & 0.7135 & 0.7163 & 0.7188 \\
$T_{\mathrm{c}}$ & $1.570710^{7}$ & $1.579210^{7}$ & $1.594710^{7}$ \\
\hline
\end{tabular}

The central temperature $T_{\mathrm{c}}$ is increasing with decreasing reaction efficiency, as expected. This is due mainly to the pp-reaction, which is the one closely linked to the luminosity of the Sun and therefore reduced screening must be balanced by higher temperature. The effect is measurable because of the rather low dependence of the reaction on temperature under solar conditions. For the same luminosity reason the flux of pp-neutrinos remains almost unchanged (Table 1; upper part). Temperature sensitive $\nu$-emission rates should increase more ( $p e p$ and hep), unless the influence of the reduced screening is dominant. Considering the ${ }^{7} \mathrm{Be}$ flux, the transition to no-screening reduces it by $2.7 \%$, but the further step to Tsytovichscreening leads to a reduction of $16.3 \%$. The ${ }^{8} \mathrm{~B}$-source, however, actually increases due to the increased temperature and its extreme temperature sensitivity. Note that in Flaskamp et al. (2000) we reported about a strongly reduced ${ }^{8} \mathrm{~B}$-flux. This result was wrong, because we erroneously had not modified the screening of the ${ }^{7} \mathrm{Be}+\mathrm{e}^{-}$ reaction, which does not appear explicitely in our nuclear network, but only as a rate relative to the ${ }^{7} \mathrm{Be}+p$-capture.

As a consequence of the modified neutrino emission rates, the predictions for the neutrino experiments change. Most interesting is the case of Super-Kamiokande, which the Tsytovich-screening model predicts to measure even more neutrinos than the standard model does, although the rate is much stronger suppressed (see Tsytovich 2000, Table 1) than the pp-reaction. However, due to the luminosity constraint and increased central temperature, the modified screening effect is overcompensated.

\section{Conclusions}

We have demonstrated that the Sun has become a sensitive laboratory to investigate the correct plasma screening for its own nuclear reactions. The high precision of the seismic Sun does not allow for deviations of more than a few percent from the original formula by Salpeter (1954). A slightly enhanced screening factor improves the agreement with the seismic sound speed profile. We emphasized that not only the central parts, where the influence of changes to the nuclear reaction rates is immediate, but also the outer layers will have a modified structure pointing out discrepancies. We have to recall, however, that this is mostly due to changing the screening of the pp-reaction. Reactions in the higher pp-chains only will not influence the models significantly. Because of these reaction rates, in contrast, the neutrino fluxes will change, not leading to the once proposed solution of the classical neutrino problem (which, as is known by now, cannot be solved by changing reaction rates only; Hata et al. 1994), but instead to even larger discrepancies, as we found for the Super-Kamiokande prediction of the Tsytovich-screening model, although this modified screening suppresses the reactions at fixed temperature.

One should keep in mind that physical pictures deriving alternative screening rates should in principle also be used to rederive the configurational effects in the equation of state and opacities to arrive at consistent physical model input. Our present work thus must remain incomplete in this respect, but we trust that the success of the standard solar model with Salpeter's screening is unlikely to be due to a conspiracy of using erroneous physics for three major model ingredients.

We believe that the Sun itself will help to settle the dispute about the true amount of screening of nuclear reactions. It has become a truely invaluable celestial laboratory for physics.

Acknowledgements. We thank H. Schlattl for his assistance in calculating the solar models and for permission to use his excellent code. A. W. acknowledges travel support from the Fulbright foundation and is grateful for the hospitality at the Princeton Observatory and the Institute for Advanced Study. He thanks A. Gruzinov, P. Krastev, and J. Bahcall for stimulating discussions. This work was supported in part by "Sonderforschungsbereich 375-95 für Astro-Teilchenphysik der Deutschen Forschungsgemeinschaft".

\section{References}

Adelberger, E. G., Austin, S. M., Bahcall, J. N., et al. 1998, Rev. Mod. Phys., 70, 1265

Antia, H., \& Chitre, S. 1999, A\&A, 347, 1000

Bahcall, J. N., Brown, L. S., Gruzinov, A., \& Sawyer, R. F. 2000a, preprint [astro-ph/0010055] 
Bahcall, J. N., Pinsonneault, M. H., \& Basu, S. 2000b, preprint [astro-ph/0010346]

Basu, S. 1998, MNRAS, 298, 719

Basu, S., \& Antia, H. M. 1995, MNRAS, 276, 1401

Basu, S., \& Antia, H. M. 1997, MNRAS, 287, 189

Basu, S., Pinsonneault, M. H., \& Bahcall, J. N. 2000, ApJ, 529,1084

Brueggen, M., \& Gough, D. O. 1997, ApJ, 488, 867

Carraro, C., Schäfer, A., \& Koonin, S. E. 1988, ApJ, 331, 565

Däppen, W., \& Nayfonov, A. 2000, ApJS, 127, 287

Degl'Innocenti, S., Dziembowski, W. A., Fiorentini, G., \& Ricci, B. 1997, Astropart. Phys., 7, 77

Fiorentini, G., Ricci, B., \& Villante, E. L. 2000, preprint [astro-ph/0011130]

Flaskamp, M., Weiss, A., \& Tsytovich, V. N. 2000, in Proceedings of the X. workshop on Nuclear Astrophysics, ed. W. Hillebrandt, \& E. Müller, MPA, 95
Grevesse, N., \& Sauval, A. J. 1998, Sp. Sci. Rev., 85, 161

Gruzinov, A. V., \& Bahcall, J. N. 1998, ApJ, 504, 996

Hata, N., Bludman, S., \& Langacker, P. 1994, Phys. Rev. D, 49(7), 3622

Salpeter, E. E. 1954, Austr. J. Phys., 7, 353

Savchenko, V. I. 1999, preprint [astro-ph/9904289]

Schlattl, H. 1999, The Sun, a Laboratory for Neutrino- and Astrophysics, Ph.D. Thesis, Techn. Univ. München

Schlattl, H., Weiss, A., \& Raffelt, G. G. 1999, Astropart. Physics, 10, 353

Shaviv, G., \& Shaviv, N. J. 2000, ApJ, 529, 1054

Shaviv, N. J., \& Shaviv, G. 1996, ApJ, 468, 433

Tsytovich, V. N. 2000, A\&AL, 356, L57

Tsytovich, V. N., \& Bornatici, M. 2000, Plasma Phys. Rep., 26,840 